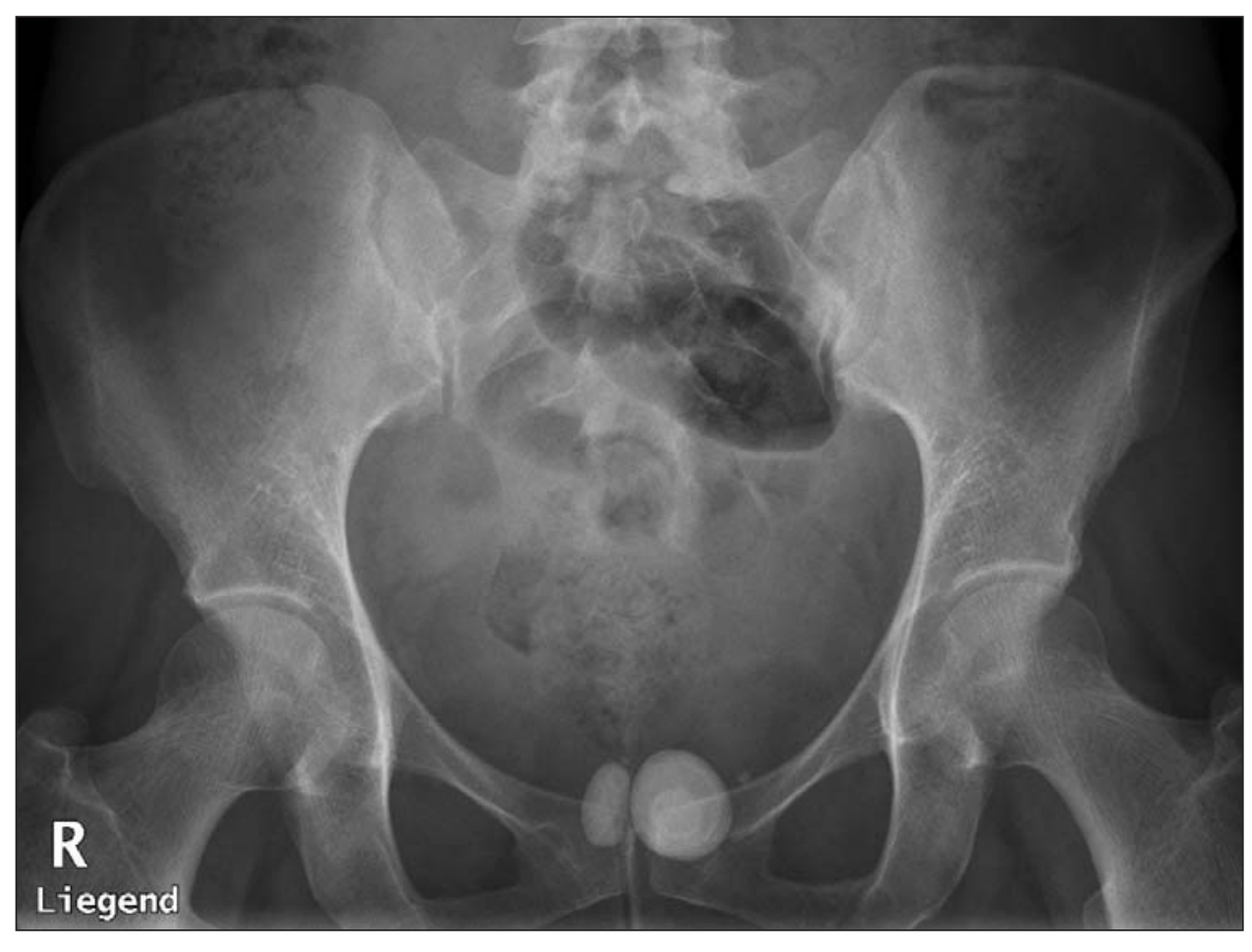

Figure 1: Radiograph showing giant calculi in a 45-year-old woman.

\title{
Giant calculi in urethral diverticula
}

$\mathrm{A}$ 45-year-old woman presented with a Io-year history of recurrent urinary tract infections. On clinical examination, we found a hard periurethral mass in the anterior vaginal wall. A radiograph of her pelvis showed giant calculi, and cystoscopy revealed an extrusion of the posterolateral distal urethra (Figure I). Urethrography and transvaginal ultrasonography confirmed 2 communicating urethral diverticula, each containing $\infty$ a stone.

The estimated prevalence of urethral diverticula in adult women is between $0.6 \%$ and $6 \%$. The condition is usually diagnosed between the ages of 20 and 50 . The cause remains largely unknown and ranges from congenital to traumatic (instrumentation, childbirth) to infectious causes. Recurrent infections and obstruction of the periurethral glands lead to abscess formation, which is presumed to rupture into the urethral lumen, thus forming the diverticulum. Associated stone formation is reported in $1.5 \%-10 \%$ of cases.

$C M A J$ invites contributions to Interesting images, a new column with a very brief but clear description of the case, the images and the main teaching point. Submit manuscripts online at http://mc.manuscriptcentral.com/cmaj.

Josef Beatrice MD Raeto T. Strebel MD Department of Urology University Hospital Zurich Zurich, Switzerland 